\section{Sequential Endoscopic Findings in Spontaneous Intramural Hematoma of the Esophagus}

Intramural hematoma of the esophagus is a rare condition, which presents clinically with substernal pain, dysphagia, or hematemesis. In this paper, we report the case of a patient who presented with a spontaneous intramural hematoma after protracted vomiting.

A 42-year-old man vomited a small amount of food after drinking. This was followed by hematemesis and substernal pain several hours later. The physical examination and hemogram, including the coagulation profile, were normal. An endoscopy carried out the following day revealed a large, purple fluctuant submucosal bulge from the left wall of the esophagus, extending from $25 \mathrm{~cm}$ distal to the incisors to the gastroesophageal junction, resulting in almost complete stenosis of the involved esophagus (Figure 1) A barium esophagogram showed an elongated, radiolucent filling defect with a smooth outline. Computed tomography showed a marked intramural hematoma of the esophageal wall, pushing the lumen to one side. The patient was treated conservatively, and was free of symptoms after three days. A subsequent endoscopy showed that the hematoma bulge seemed to rupture into the lumen with a remaining blood clot, and had lost its mucosal layer (Figure 2). On the tenth day of hospitalization, the bulge had disappeared, and there was a longitudinal depressed lesion over the involved esophagus. One month later, esophagoscopy revealed spontaneous resolution with normal mucosa, and the pathology of a biopsy taken showed mild submucosal fibrosis (Figure 3 ).

Spontaneous intramural hematoma of the esophagus was first described by Marks and Keet in 1968 (1), and the incidence is relatively low. The esophagogram shows a sharply-defined filling defect, or a sign of double-barrelled esophagus (2). Fiberoptic esophagoscopy provides a definitive diagnosis. Computed tomography can reveal marked eccentric thickening of the esophageal wall, and is helpful in excluding cardiovascular diseases. The treatment is conservative, and surgery should be avoided (3). The pathogenesis is controversial (4). Some authors have considered that complete healing of an intramural hematoma took a long time, and suggested that close follow-up was required, but no images or data have previously been available (5). In our case, the course seemed benign and self-limited.

\section{M.-T. Hsu, X.-Z. Lin, T.-T. Chang, J.-S. Shin, C.-Y, Chen,}

B.-S, Sheu

Division of Gastroenterology, Dept. of Medicine, National Cheng Kung University Hospital, Tainan, Taiwan

\section{References}

1. Marks IN, Keet AD. Intramural rupture of the esophagus. $\mathrm{Br}$ Med J 1968; 3; 536-7.

2. Barone JE, Robilotti JG, Comer JV. Conservative treatment of spontaneous intramural perforation (or intramural hematoma) of the esophagus. Am J Gastroenterol 1980; 74: 165-7.

3. Yeoh NTL, MeNicholas T, Rothwell-Jackson RL, et al. Intramural rupture and intramural haematoma of the esophagus. Br J Surg 1985; 72: 958-60.

4. Spiller RC, Catto JV, Kane SP, Spontaneous dissecting intramural hematoma to the esophagus: a rare cause of haematemesis and dysphagia. Endoscopy 1981: 13: 128-30.

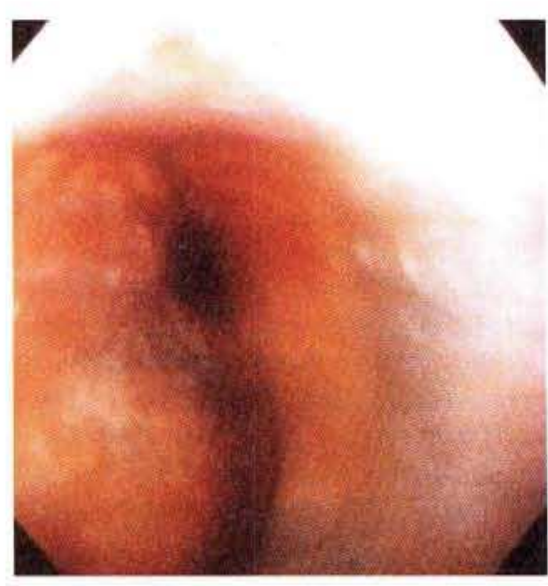

Figure 1: Endoscopic finding at the initial examination.

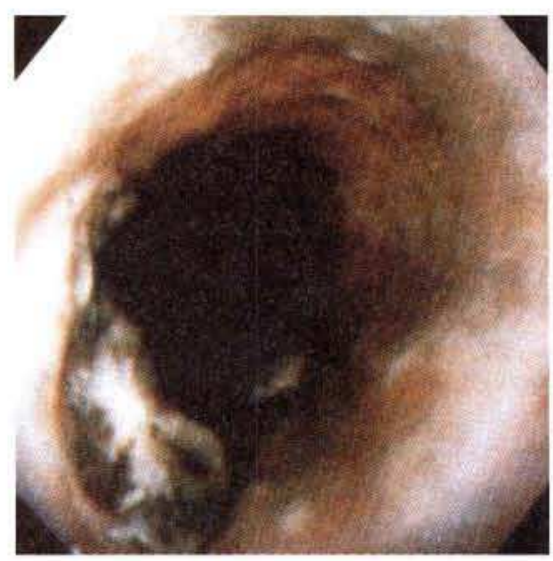

Figure 2: Endoscopic findings five days later.

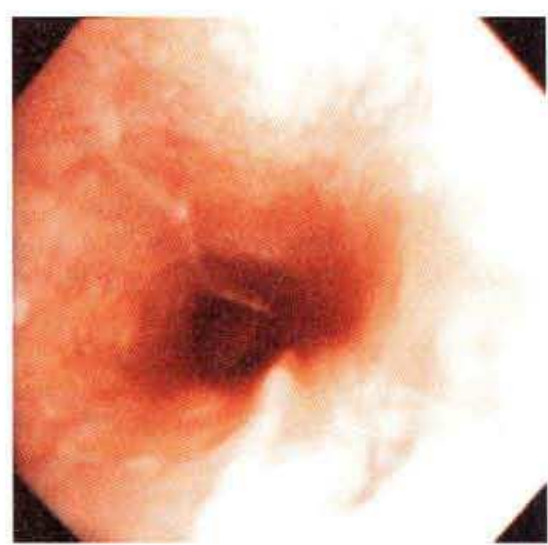

Figure 3: Endoscopic findings one month later

5. Furukawa H, Hara T, Taniguchi T, et al. A case of spontaneous intramural hematoma of the esophagus. Gastroenterol Jpn $1993 ; 28: 81-7$.

Corresponding Author

X.-Z. Lin, M.D.

Division of Gastroenterology

Dept, of Medicine

National Cheng Kung University Hospital

138 Sheng Li Road

Tainan 704

Taiwan

Fax: $+886-6-2347270$ 